\title{
Editorial
}

\section{Partial Fractional Equations and Their Applications}

\author{
Abdon Atangana, ${ }^{1}$ Hossein Jafari, ${ }^{2}$ Samir B. Belhaouari, ${ }^{3}$ and Mustafa Bayram ${ }^{4}$ \\ ${ }^{1}$ Institute for Groundwater Studies, Faculty of Natural and Agricultural Sciences, University of the Free State, \\ Bloemfontein 9300, South Africa \\ ${ }^{2}$ Department of Mathematics, Faculty of Sciences, University of Mazandaran, P.O. Box 47416-95447, Babolsar, Iran \\ ${ }^{3}$ Department of Mathematics and Applied Mathematics, Faculty of Sciences and Technology, Alfaisal University, \\ P.O. Box 50927, Riyadh 11533, Saudi Arabia \\ ${ }^{4}$ Department of Mathematical Engineering, Yildiz Technical University, Davutpasa, 34210 Istanbul, Turkey
}

Correspondence should be addressed to Abdon Atangana; abdonatangana@yahoo.fr

Received 11 March 2015; Accepted 11 March 2015

Copyright (C) 2015 Abdon Atangana et al. This is an open access article distributed under the Creative Commons Attribution License, which permits unrestricted use, distribution, and reproduction in any medium, provided the original work is properly cited.

In the past years, many physical problems in almost all branches of sciences and engineering were with success modelled using the concept of fractional order derivative. This subject has therefore fascinated consideration of many researchers around humankind. Particularly, the advantages of the concept of fractional calculus and their associated models via differential partial differential equations linking fractional order integrodifferential operators and their appliances have been previously intensively investigated with great success during the last years.

Despite the fact that the exploration of the phenomena is portrayed by interface of many organisms, the microreplications play an important role, and as result the computers turn out to be progressively scientific apparatus. The main reason for this special issue was to report and review the latest progress in the areas of partial differential equations. This special issue included in particular the following:

(i) fractional partial differential equations and their applications in sciences and engineering,

(ii) modelling and simulation of real world phenomena with partial differential equations,

(iii) analytical and numerical methods for partial differential equations,

(iv) new applications of iterations method,

(v) anomalous diffusion.
We have received many submissions, however, to keep the standard high only few were accepted for publications. This special issue includes papers on dissimilar features, for instance, analysis of Sturm-Liouville Eigen-problems, numerical methods and analysis, iterative methods, fractional boundary value problems, analytical method like Lie symmetry analysis, and integral transform operators.

Evidently, it is not imaginable to satisfactorily distinguish in this special issue all the strategy of up-to-date exploration in fractional partial differential equations with their applications; however, we assumed that this special isssue contents both hypothetical investigate and significant progress including contemporary challenging problems, new ideas, and open problems.

\section{Acknowledgment}

We acknowledge all the contributors to this special issue for their participations.

Abdon Atangana

Hossein Jafari

Samir B. Belhaouari

Mustafa Bayram 


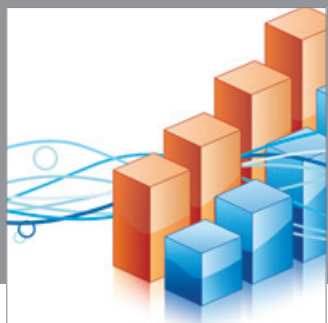

Advances in

Operations Research

mansans

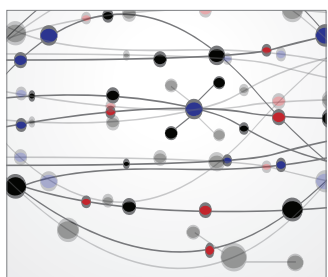

The Scientific World Journal
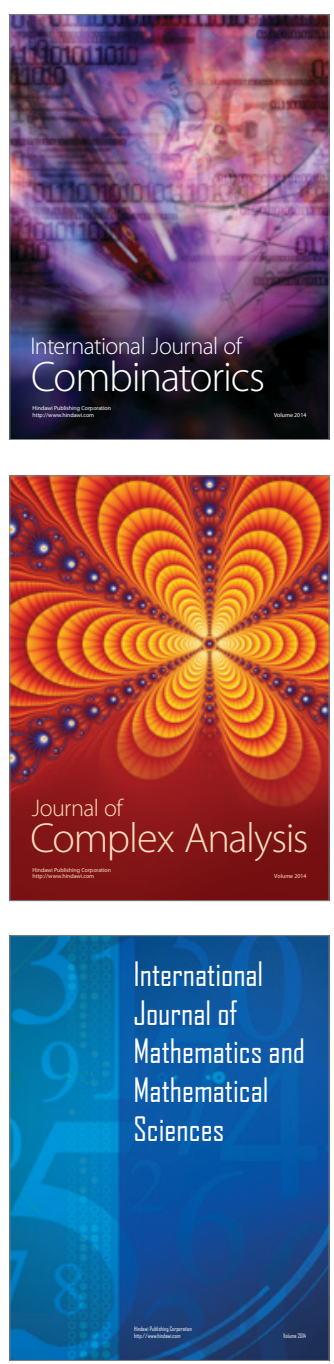
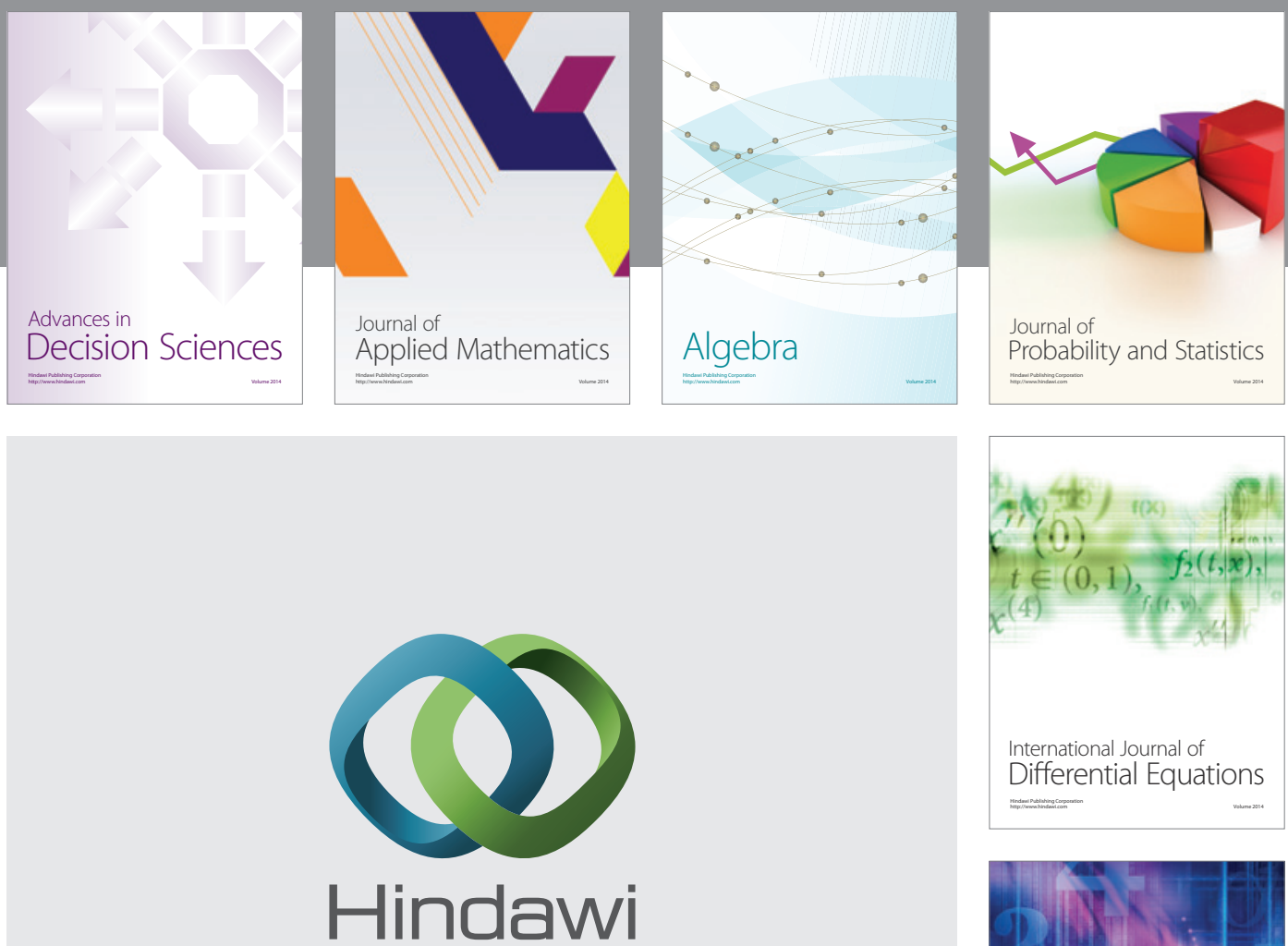

Submit your manuscripts at http://www.hindawi.com
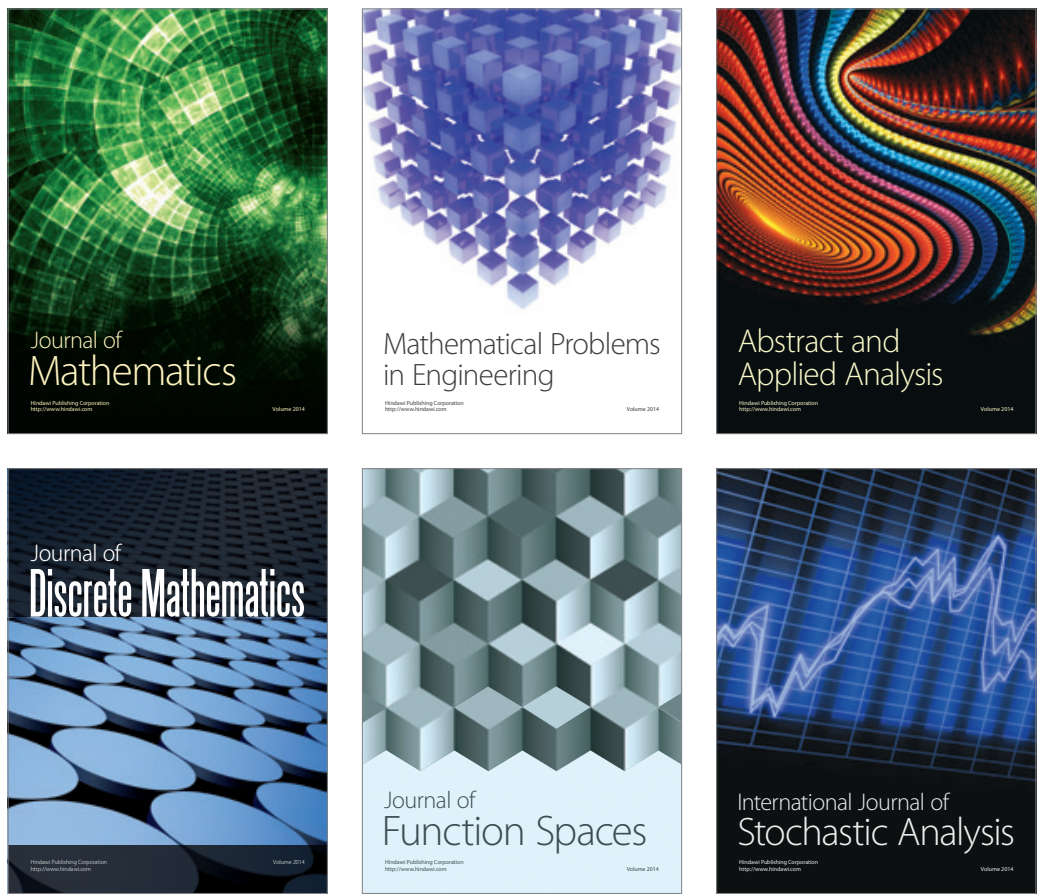

Journal of

Function Spaces

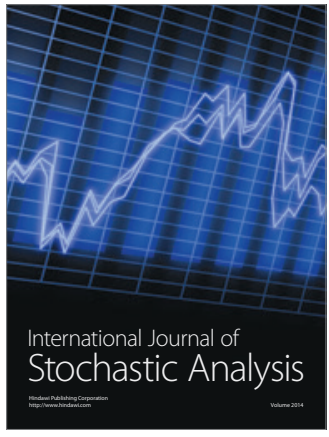

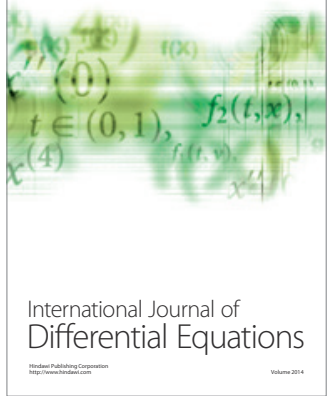
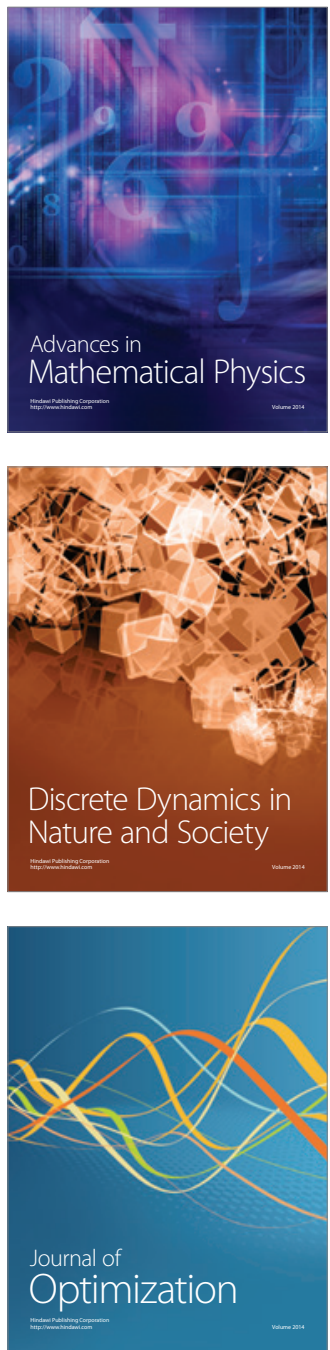